\title{
Aufklärung versus Angstmacherei: Die Darstellung der Atomkatastrophe in der deutschsprachigen und französischen Kinder- und Jugendliteratur
}

Hildegard Haberl

\section{(2) OpenEdition \\ Journals}

Édition électronique

URL : https://journals.openedition.org/allemagne/2266

DOI : 10.4000 /allemagne.2266

ISSN : 2605-7913

Éditeur

Société d'études allemandes

Édition imprimée

Date de publication : 1 juillet 2020

Pagination : 115-128

ISSN : 0035-0974

\section{Référence électronique}

Hildegard Haberl, „Aufklärung versus Angstmacherei: Die Darstellung der Atomkatastrophe in der deutschsprachigen und französischen Kinder- und Jugendliteratur", Revue d'Allemagne et des pays de langue allemande [Online], 52-1 | 2020, Online erschienen am: 06 Juli 2021, abgerufen am 18 Februar 2022. URL: http://journals.openedition.org/allemagne/2266 ; DOI: https://doi.org/10.4000/allemagne. 2266 


\title{
Aufklärung versus Angstmacherei: Die Darstellung der Atomkatastrophe in der deutschsprachigen und französischen Kinder- und Jugendliteratur
}

- Hildegard Haberl*

\begin{abstract}
„Alors, c'est quoi, Tchernobyl? Un signe? Ou une gigantesque catastrophe technologique, sans commune mesure avec aucun événement du passé?"

Svetlana Alexievitch(1)

"Ich schreibe lieber über andere Themen als über Katastrophen. Aber leider Gottes sind eben diese Themen oft mit der Gefahr, die von Katastrophen ausgehen, verbunden."

Gudrun Pausewang ${ }^{(2)}$
\end{abstract}

Die deutschsprachige Kinder- und Jugendliteratur (KJL) hat vergleichsweise früh „Klassiker der ökokritischen Literatur“(3) hervorgebracht, die die Gefahr der Atomwaffennutzung und das Szenario eines möglichen Supergaus ins Zentrum der Handlung stellen. Es ist vom „Paradigmenwechsel der 1970er Jahre“(4) die Rede, wenn über Ökologie in der Kinder- und Jugendliteratur gesprochen wird. Dabei ist die KJL nicht von der allgemeinen Literatur (oder auch „All-Age-Literatur ${ }^{\text {“(5) }}$ ) zu trennen:

* Maîtresse de conférences en études germaniques, Université de Caen Normandie, ERLIS EA 4254.

1 Svetlana Alexievitch, La supplication. Tchernobyl, chronique du monde après l'apocalypse (1997), traduit du russe par Galia Ackerman et Pierre Lorrain, Paris, J’ai lu, 2009, S. 31.

2 Gudrun Pausewang in einem Online-Interview: epilog.de/ich-moechte-warnen-ein-gespraech-mitder-autorin-gudrun-pausewang-12-04-2006/ (26.08.2019).

3 Berbeli Wanning, Anna Stemman, „Ökologie in der Kinder- und Jugendliteratur“, in: Gabriele Dürвеск und Urte Sтовве (Hg.), Ecocriticism. Eine Einführung, Köln, Böhlau, 2015, S. 258-270, hier S. 263.

4 Anna Stemman, „Genretransgressionen und hybride Erzählstrategien in ökologischen Krisenszenarien der Kinder- und Jugendliteratur“, in: Evi ZemaneK (Hg.), Ökologische Genres. NaturästhetikUmweltethik - Wissenspoetik, Göttingen, Vandenhoeck \& Ruprecht, 2018, S. 281-295.

5 Leonie Jankowsкi, Dystopieromane der Kinder-und Jugendliteratur als All-Age-Lektüre: am Beispiel von Gudrun Pausewangs „Die Wolke“, Saarbrücken, AV Akademikerverlag, 2017. 
„Der Themenkomplex Umweltschutz, Ökologie, Nachhaltigkeit - bzw. die Bedrohung und Zerstörung des ökologischen Gleichgewichts findet im Verlauf der 1970er Jahre Eingang in die moderne Kinder- und Jugendliteratur, d.h. signifikanterweise genau zu dem Zeitpunkt, als man anfängt, dieses Thema auch diskursiv in der gesellschaftspolitischen Öffentlichkeit zu verhandeln und es im Zuge dieses Diskurses auch von allgemeinliterarischen Erzählungen aufgegriffen wird. [...]“(6).

Was die atomare Bedrohung angeht, hat der Österreicher Karl Bruckner (19061982) sehr früh die Gefahren der militärischen Nutzung der Atomtechnologie in seinem Roman Sadako will leben, der 1961 erschienen ist, thematisiert ${ }^{(7)}$. Es geht in diesem Text vor allem um die Auswirkungen des Atombombenabwurfs auf die Zivilbevölkerung, genauer die Darstellung der Strahlenkrankheit, an der die Hauptfigur Sadako stirbt. Bruckners Buch wurde seither in siebzig Sprachen übersetzt und über zwei Millionen Mal verkauft. Ebenso erfolgreich war und ist Gudrun Pausewangs Bestseller Die Wolke. Jetzt werden wir nicht mehr sagen können, wir hätten von nichts gewußt ${ }^{(8)}$, der ein Jahr nach der atomaren Katastrophe von Tschernobyl im April 1986 erschienen ist ${ }^{(9)}$. Sowohl Bruckners Roman als auch Die Wolke haben den deutschen Jugendliteraturpreis erhalten und Generationen Jugendlicher für die Gefahren der Atomwaffen bzw. die Risiken eines Reaktorunfalls sensibilisiert. Die Verleihung dieses Preises an Pausewang hat richtiggehend einen Literaturskandal ausgelöst und, wie Gabriele von Glasenapp festhält, war kaum ein jugendliterarisches Werk umstrittener und wirkungsvoller als dieser Roman, da Teile der regierenden CDU sich gegen die Verleihung des Preises an Gudrun Pausewang ausgesprochen hatten und aktiv versuchten, in das Preisverfahren einzugreifen $^{(10)}$. Kritisiert wurde vor allem die negative Darstellung von Politikern im Text, denen ein Nichtwahrhabenwollen des Risikos, Verantwortungslosigkeit und

6 Gabriele von Glasenapp, „Akopalypse now! Formen und Funktionen von Utopien und Dystopien in der Kinder- und Jugendliteratur“, in: Hans-Heino Ewers, Gabriele von Glasenapp, Claudia Maria Pecher (Hg.), Lesen für die Umwelt. Umwelt und Umweltschutz in der Kinder-und Jugendliteratur, Baltmannsweiler, Schneider Verlag Hohengehren GmbH, 2013, S. 67-86. Auch Natalie PoHL sieht einen starken soziokulturellen Einschnitt und Wendepunkt der Umweltgeschichte in den 70er Jahren in: „Une histoire du temps présent nucléaire: Le mouvement anti-nucléaire franco-allemand dans la région du Rhin supérieur", in: Emmanuel Droit, Hélène Miard-Delacroix, Frank Reicherzer (Hg.), Penser et pratiquer l'histoire du temps présent, Villeneuve d'Ascq, Presses universitaires du Septentrion, 2016, S. 27-36.

7 Karl Bruckner, Sadako will leben (1961), Wien, Wien G\&G Verlagsgesellschaft mbH, 2005.

8 Gudrun Pausewang, Die Wolke. Jetzt werden wir nicht mehr sagen können, wir hätten von nichts gewußt (1987), Otto Maier Ravensburg (Ravensburger Junge Reihe), 2006.

9 Gudrun Pausewang hat sich in weiteren Büchern mit der atomaren Bedrohung oder anderen Formen der Umweltzerstörung auseinandergesetzt: Die letzten Kinder von Schewenborn oder...sieht so unsere Zukunft aus?, Ravensburger Buchverlag, 1983 (für Kinder ab 12 Jahren); Es ist doch alles grün, 1994 (für Kinder ab 9 Jahren); Die Kinder in der Erde, Ravensburger Buchverlag, 1988, Illust. Annegret Fuchshuber (für Kinder ab 6 Jahren). 2012 erschien der Roman Noch lange danach, in dem sich Gudrun Pausewang erneut mit den möglichen Folgen eines Super-GAUs beschäftigt.

10 G. v. Glasenapp, „Akopalypse now! (Anm. 6), S. 67-86, hier S. 76. Weiters: Susan Tebbutt, „Wie Skandale entstehen und wohin sie führen können: Der Skandal um Gudrun Pausewangs Antiatomroman Die Wolke (1987)“, in : Stefan Neuhaus und Johann Holzner (Hg.), Literatur als Skandal. Fälle - Funktionen - Folgen, Göttingen, Vandenhoeck \& Ruprecht, 2007, S. 559-567. 
hohle Phrasendrescherei ${ }^{(11)}$ vorgeworfen wird. In jedem Fall hat der Medienwirbel um diese Preisverleihung zur großen Bekanntheit des Romans maßgeblich beigetragen $^{(12)}$. 2006 wurde dieser dann von Gregor Schnitzler verfilmt, 2008 erschien eine Comic-Adaption von Anika Hage. Der Roman wurde in dreizehn Sprachen und 1996 von François Mathieu auch ins Französische übersetzt ${ }^{(13)}$.

„Die andauernde Wirkungsmächtigkeit des Romans manifestiert sich nicht zuletzt darin, dass die deutsche Atomindustrie die Verfilmung des Romans zu verhindern suchte und, da dies nicht möglich war, doch wenigstens die Benennung genauer Örtlichkeiten (konkret ging es um das AKW in Grafenrheinfeld) zu unterbinden (im Film trägt das Kernkraftwerk schließlich den fiktiven Namen Markt Ebersberg). Heute, im Jahre 2013, ist das Werk, wie Orwells Dystopie 1984, ein jugendliterarischer Klassiker (wenngleich er im Zuge der Atomkatastrophe von Fukushima noch einmal kurzfristig auf den Bestsellerlisten auftauchte), der vor allem etwas darüber aussagt, auf welche Weise in den späten 1980er Jahren die Katastrophe von Tschernobyl in jugendliterarischen Werken verhandelt wurde“(14).

Auf diese Art und Weise der Verhandlung der Atomkatastrophe möchte ich in meinem Beitrag näher eingehen und zum Vergleich einen jüngeren französischen Jugendroman von Aurélie Wellenstein, La fille de Tchernobyl, heranziehen ${ }^{(15)}$. Wie wird die Katastrophe erzählt und gestaltet, damit sie für Jugendliche (und Erwachsene) fassbar wird? Wellenstein ist 1980 geboren, hat also einen ganz anderen biographischen Hintergrund als die 91-jährige Gudrun Pausewang, die sich, vom Zweiten Weltkrieg geprägt ${ }^{(16)}$, sehr früh gegen die Gefahr der Atomkraftnutzung in der Friedensbewegung engagiert hat ${ }^{(17)}$. Pausewang gehört also zu der Generation von Schriftstellerinnen und Schriftstellern, die bereits in den frühen achtziger Jahren im Kontext von Nachrüstungsdebatte, atomarer Bedrohung und Waldsterben sowie in Auseinandersetzung mit dem Zweiten Weltkrieg apokalyptische Szenarien geschaffen haben ${ }^{(18)}$. Pausewangs Buch ist vom Erscheinungsdatum her betrachtet eine „NearFuture-Fiction“, wohingegen Wellensteins Text eine realistische Erzählung nach der Katastrophe darstellt, die aber auch Fantasy-Elemente enthält.

Für mich als Auslandsgermanistin ist es überraschend festzustellen, dass in Frankreich - einem Land mit achtundfünfzig Reaktoren, die zweiundsiebzig Prozent des Strombedarfs decken - erst die Katastrophe von Fukushima zu einer zunehmenden

11 Wie zum Beispiel „Bald kommt alles wieder in Ordnung“ (Die Wolke [Anm. 8], S. 90).

12 Regina Pantos, „Störfälle. Der Streit um die Verleihung des Deutschen Jugendliteraturpreises 1988“, kjl\&m, 61/4 (2009), S. 19-22.

13 Gudrun Pausewang, Le nuage, trad. par. François Mathieu, Paris, Pocket Jeunesse, 2005.

14 G. v. Glasenapp, „Akopalypse now! (Anm. 6), S. 76.

15 Aurélie Wellenstein, La fille de Tchernobyl, Paris, Magnard, 2016 (8-12 ans).

16 Cf. ihre autobiographischen Bücher über ihre Kindheit und Jugend zusammengefasst in Gudrun PAUSEWANG, Rosinkawiese - damals und heute. Die Rosinkawiesen-Trilogie in einem Band, München, dtv, 2004. Oder auch Gudrun Pausewang, Ich war dabei. Geschichten gegen das Vergessen. Eigene und fremde Erlebnisse aus der Nazi-Zeit, Düsseldorf, Sauerländer Verlag, 2004.

17 Uwe Jahnke, Gudrun Pausewang, Leben und Werk, Ravensburg, Ravensburger, 2010. 2017 hat Gudrun Pausewang auf der Frankfurter Buchmesse den „Sonderpreis des Deutschen Jugendliteraturpreises" für ihr Lebenswerk erhalten.

18 Cf. Axel Goodbody, „Literatur und Ökologie: Zur Einführung“, in: Axel Goodbody (Hg.), Literatur und Ökologie, Amsterdam/Atlanta (GA), Rodopi, 1998, S. 11-40, hier S. 24 sq. 
literarischen Auseinandersetzung mit Tschernobyl geführt hat ${ }^{(19)}$. Bis dahin gab es keine vergleichsweise „einflussreiche“ politisch engagierte kritische Kinder- und Jugendliteratur ${ }^{(20)}$ wie die von Bruckner und Pausewang, auch wenn es falsch wäre zu behaupten, dass nichts dazu erschienen wäre ${ }^{(21)}$. Dieses ,Fehlen' würde dennoch die Hypothese der Soziologin Sezin Topçu stützen, die in ihrem Buch über La France nucléaire. L'art de gouverner une technologie contestée behauptet, dass der weltweit schwerste Unfall in der zivilen Nutzung der Atomenergie die Antiatombewegung in Frankreich paradoxerweise eher gebremst als neu belebt hätte ${ }^{(22)}$ : „Le défilé antinucléaire organisé le 11 mai à Paris rassemble à peine trois mille manifestants, alors que les mobilisations font rage dans les pays voisins (Allemagne, Angleterre, Autriche, Italie, Suisse...), avec des dizaines de milliers de manifestants, voire cent cinquante mille à Rome ce même 10 mai $1986^{\text {“(23). }}$.

In einem aktuellen Spiegel-Artikel wird von einem dreifachen französischen Atomkonsens gesprochen, zu dem auch die Tatsache gezählt wird, dass Frankreich seine eigene Größe und Macht noch immer durch seine Atomtechnik garantiert sieht, woran auch die Bürgerinnen und Bürger in der Mehrheit offenbar nicht rütteln wollen $^{(24)}$.

Aurélie Wellensteins Roman, der 2016 erschienen ist, kann also als marginalisierte Stimme im gesellschaftlich dominanten Diskurs der Atomkraftbefürwortung gelesen werden $^{(25)}$. Der Text ist intertextuell stark von Swetlana Alexijewitschs Dokumentarroman Tschernobyl. Eine Chronik der Zukunft $(1997)^{(26)}$ geprägt, der wiederum eine Collage aus Zeugenberichten von betroffenen Menschen aus Tschernobyl ist: von Frauen und Müttern der Feuerwehr- und Rettungsmänner, Arbeitern des Atomkraftwerks,

$19 \mathrm{Zu}$ diesem jüngeren Interesse an der Atomkatastrophe würde ich auch den Erfolg der amerikanischbritischen Fernsehserie Chernobyl von Craig Mazin zählen (HBO, 2019).

20 Ich spreche hier natürlich nicht von den zahlreichen pädagogischen Filmen und Dokumenten, die ab der zweiten Hälfte der 70er Jahre den technologischen Fortschritt der Atomindustrie beweisen sollen. Ich denke dabei an kritische Literatur wie das Comic Plogoff von Delphine Le Lay und Alexis Horrellou, das 2013 bei Delcourt erschienen ist und den Widerstand des bretonischen Dorfes Plogoff gegen den Bau eines Atomkraftwerkes in den Jahren 1974-1981 in Erinnerung ruft.

21 Das belgische Internetnetzwerk „Réseau IDée - Information et Diffusion en éducation à l'environnement“, das seit 1989 besteht und sich mit der Pädagogik von Umweltthemen befasst, stellt interessante Literaturlisten von Sachbüchern, Comics und Belletristik online zur Verfügung, www.reseau-idee.be/ outils-pedagogiques/fiche.php?\&media_id=2208 (15.11.2019).

22 Sezin Topçu, La France nucléaire. L’art de gouverner une technologie contestée, Paris, Seuil, 2013.

23 Ebd., S. 153.

24 Georg Blume, „Drei Gründe, warum Frankreich nicht von der Atomkraft lässt“, Der Spiegel, 03.01.2020, www.spiegel.de/politik/ausland/atomkraft-in-frankreich-drei-gruende-warum-das-land -6-neue-akw-plant-a-1301888.html (05.01.2020); cf. auch Sandra TAuER, Störfall für die gute Nachbarschaft? Deutsche und Franzosen auf der Suche nach einer gemeinsamen Energiepolitik (1973-1980), Göttingen, V\&R unipress, 2012.

25 Weitere Ausnahmen sind: Lucile Bordes, 86, année blanche, Paris, Liana Levi, 2016, in dem die 1971 geborene Autorin ihre Erinnerungen an die Katastrophe von Tschernobyl verarbeitet oder auch Alexandra Koszely K, À crier dans les ruines, Paris, Éditions Aux forges de Vulcain, 2019.

26 Swetlana Alexijewitsch, Tschernobyl. Eine Chronik der Zukunft (1997), übersetzt von Ingeborg Kolinko und Ganna-Maria Braungardt, Frankfurt am Main, Suhrkamp, 2019; auf Französisch: S. Alexievitch, La supplication. Tchernobyl, chroniques du monde après l'apocalypse (Anm. 1). 
Soldaten, Ärzten usw. Im Jahr 2015 hat Alexijewitsch für ihr literarisches Werk den Nobelpreis erhalten $^{(27)}$.

Welche narrativen Besonderheiten weisen nun diese Katastrophendarstellungen in der Kinder- und Jugendliteratur auf? Im ersten Teil meines Beitrages möchte ich die weiblichen Hauptfiguren der zwei Jugendromane vorstellen und dabei auch auf die Verknüpfung von faktualem und fiktionalem Modus eingehen. Im zweiten Teil werden die zerstörten Räume analysiert und im abschließenden Teil soll gezeigt werden, inwieweit diese Romane Erinnerungsarbeit leisten und gleichzeitig Aufklärung einfordern ${ }^{(28)}$. Anders gesagt, soll es um die Frage nach den erzieherischen Absichten dieser Texte gehen. Sind sie reine „Angstmacherei“, wie es Gudrun Pausewang vorgeworfen wurde, oder hat das Setting der „Katastrophe“, das nicht neu ist, sondern eine lange literarische Tradition hat ${ }^{(29)}$ und in der internationalen Umweltbewegung eine zentrale Rolle spielt ${ }^{(30)}$, noch andere Funktionen?

\section{Kinder der Katastrophe: Hibakushas und mutige Mädchen}

„,Ich übertreibe?', Almut lächelte. ,Du solltest dir mal ein Buch über Hiroshima besorgen. Die Überlebenden dort und wir - und alle, die vielleicht noch dazukommen: Wir sind die Aussätzigen des zwanzigsten Jahrhunderts“" (Pausewang, Die Wolke, S. 150).

Um zu erklären, woher das Wort „Hibakusha“ stammt, muss zuerst noch einmal kurz auf Bruckners Sadako will leben verwiesen werden, in dessen Nachfolge Pausewang mit ihrer Figurengestaltung einzuordnen ist. Sadako ist eine historische Figur. Es handelt sich dabei um das japanische Mädchen Sadako Sasaki, das am 7. Januar 1943 geboren wurde. Mit zwei Jahren erlebte sie den Atombombenabwurf auf Hiroshima und Nagasaki, an dessen Folgen sie zehn Jahre später erkrankte und am 25. Oktober 1955 starb. Sie wurde zur weltweit bekanntesten Hibakusha, d.h. zu einer Überlebenden des Atombombenabwurfs. Bruckner wurde durch einen Bericht des Journalisten, Friedensforschers und Autors des Atomstaats ${ }^{(31)}$, Robert Jungk (19131994), auf die Geschichte dieses Mädchens aufmerksam ${ }^{(32)}$, das, nachdem es an der Strahlenkrankheit erkrankte, tausend Papierkraniche falten wollte, um durch die

27 Alexijewitschs Text scheint mir zentral für eine veränderte Rezeption der Katastrophe von Tschernobyl in Frankreich, worauf ich jedoch an dieser Stelle nicht genauer eingehen kann.

28 Cf. Peter Utz, Kultivierung der Katastrophe. Literarische Untergangsszenarien aus der Schweiz, München, Wilhelm Fink Verlag, 2013. Utz formuliert 10 Thesen hinsichtlich der Katastrophendarstellung in der Literatur, zu der auch die Erinnerungsarbeit gehört. Vgl. auch Benjamin BüHLER, Ecocriticism. Eine Einführung. Grundlagen - Theorien - Interpretationen, Stuttgart, Metzler, 2016, S. 169-179 (Katastrophe, Störung, Risiko).

29 Cf. A. Goodbody, „Literatur und Ökologie“ (Anm. 18), S. 11-40, hier S. 33-34.

30 Ebd., S. 34. Siehe auch Gunter E. Grimm, Werner Faulstich und Peter Kuon (Hg.), Apokalypse. Weltuntergangsszenarien in der Literatur des 20. Jahrhunderts, Frankfurt am Main, Suhrkamp, 1986; Klaus Vondung, Die Apokalypse in Deutschland, München, dtv, 1988.

31 Robert Jungk, Der Atomstaat. Vom Fortschritt in die Unmenschlichkeit, München, Kindler, 1977 (3. Aufl.).

32 Cf. auch Yôko Koyama-Siebert, „Atomare Kinder- und Jugendliteratur in Deutschland. Ein Vergleich“, Literaturkritik.de, Nr. 4, April 2016, literaturkritik.de/public/rezension.php?rez_id=21859 (04.09.2019). 
Kraft dieses Symbols wieder gesund zu werden. Zur „Hibakusha“ wird auch Pausewangs Hauptfigur in Die Wolke, der einen fiktiven GAU (Größter anzunehmender Unfall) und dessen Folgen mitten in Deutschland, genauer in Osthessen/Unterfranken im Städtchen Schlitz, behandelt.

Die Katastrophe ereignet sich in Pausewangs Roman an einem ganz normalen Schultag, an dem plötzlich die Alarmglocke ertönt. Es ist ein besonderer Alarm - ein ABCAlarm -, der bei einer atomaren, biologischen und chemischen Bedrohung ertönt. Das etwa achtzig Kilometer entfernte Atomkraftwerk wird zum Störfall, genauer gesagt zum nicht mehr beherrschbaren Störfall ${ }^{(33)}$. Die heile Welt kippt in ein Katastrophenszenario um. Alle Schüler wollen nach Hause. Janna-Berta, die 14-jährige Hauptfigur, muss sich um ihren kleinen Bruder Uli kümmern, der zu Hause auf sie wartet. Die anderen Geschwister und ihre Eltern sind an diesem Tag in Schweinfurt, in der Nähe des Reaktors, beschäftigt. Sie kann mit einem Schulkollegen von der Schule in ihr Dorf zurückfahren und im Auto erinnert sie sich nun an andere Störfälle und an das Antiatomengagement ihrer Eltern nach Tschernobyl, wobei hier auf den Generationenkonflikt hinzuweisen ist, der die technologiefreundliche Großelterngeneration (der Nachkriegsjahre) der skeptischen 68er-Generation gegenüberstellt:

„Im Fond diskutierten sie jetzt über die Art des Grafenrheinfelder Reaktors und darüber, was in einem solchen Reaktor passiert sein konnte. Immer wieder fielen die Wörter ,Tschernobyl', ,Harrisburg', ,Brennstäbe‘, ,Kühlwasser' und ,Druckbehälter'. [...] Nach Tschernobyl war sie mit ihren Eltern auf mehreren Demonstrationen gewesen. Sie erinnerte sich noch gut daran. Damals hatte es den Riesenkrach gegeben zwischen den Eltern und den Großeltern: Oma Berta und Opa Hans-Georg meinten, ohne Atomkraft gehe es einfach nicht mehr, die gehöre nun mal zum modernen Leben wie das Auto oder der Fernseher, und dass da in Tschernobyl was schiefgelaufen sei, das habe mit den deutschen Atomkraftwerken überhaupt nichts zu tun. Außerdem: Mit Demonstrationen bewege man gar nichts, das seien nur Tummelplätze für Träumer und Chaoten“ (Pausewang, Die Wolke, S. 17).

Janna-Berta kann kurz mit ihrer Mutter telefonieren, die ihr die Flucht nach Bad Hersfeld rät. Die Situation wird chaotisch und anarchisch. Niemand hält sich mehr an Regeln. Jeder denkt nur an sein Fortkommen, denn alle haben Angst vor der Wolke, die sich vom Katastrophengebiet Richtung Westen bewegt. Auf tragische Weise wird der kleine Bruder auf einer Landstraße von einem Auto erfasst und ist sofort tot. JannaBerta steht unter Schock und wird von einer jungen Familie mitgenommen. Sie muss den Leichnam ihres Bruders liegen lassen. Die Züge sind überfüllt und Janna-Berta läuft zurück ins Katastrophengebiet, inmitten eines verseuchten, wolkenbruchartigen Regens. Studenten in einem Kleinbus lesen sie vollkommen durchnässt und verstört neben der Autobahn auf. Sie wollen über die Grenze in den Osten (Thüringen), werden dort jedoch abgewiesen. Sie beschließen, Richtung Norddeutschland weiterzufahren. Janna-Berta will nicht in den Norden, auch wenn dort ihre Tante Helga, die Schwester ihres Vaters, wohnt, sondern zu ihrer Tante Almut und deren Mann Reinhard, die sie in der Nähe vermutet. Sie steigt aus, setzt ihre Suche fort und bricht wenig später erneut zusammen. Sie wird in ein Notkrankenhaus in Herleshausen (Hessen) gebracht. Von der Einteilung des Katastrophengebiets in Sperrzonen hört sie durch ein Gespräch von zwei Krankenschwestern. 
„Nachdem Janna-Berta eine Weile zugehört hatte, begriff sie, dass es offenbar drei Sperrzonen gab: Sperrzone EINS war das Umfeld des Reaktors von Grafenrheinfeld. Dort, so hieß es, habe niemand überlebt. Das Terrain war für eine unabsehbare Zeit unbewohnbar geworden. Auch die Zone ZWEI, die sich daran anschloss - sie reichte von Bad Brückenau bis Coburg -, war noch stark verseucht und würde für Jahre gesperrt bleiben. Nur die Flüchtlinge aus Zone DREI konnten hoffen, in ein paar Monaten wieder heimkehren zu dürfen“" (Pausewang, Die Wolke, S. 87).

Im Nothospital erfährt sie vom Tod ihrer Eltern, ihrer geliebten Großmutter Jo und ihres zweiten Bruders Kai. Nach einem längeren Aufenthalt wird sie von ihrer Tante Helga nach Hamburg geholt, bei der sie sich jedoch nicht geborgen fühlt. Janna-Berta hält es in Hamburg nicht mehr aus und macht sich auf den Weg zu ihrer Tante Almut, die als Betroffene dabei ist, in Wiesbaden-Bierstadt ein Hibakusha-Zentrum aufzubauen. Am Beispiel der Figur der Hibakusha zeigt sich die literarische Herausbildung einer solidarischen Gruppe, auf die auch Peter Utz hinweist ${ }^{(34)}$ : In der Katastrophe formt sich ein Kollektiv der Opfer, „die eine Kernzelle von sozialer und nationaler Kohäsion bilden, die durch die gemeinsam geteilte Erfahrung des Schreckens gestärkt wird“(35). Eine neue Familie entsteht für Janna-Berta um Almut und Reinhart, dessen Vater, zwei adoptierten Kindern und deren Großmutter. Endlich kehrt Janna-Berta in die Sperrzone 3 zurück. Sie kann nun endlich ihren Bruder begraben. In der Begräbnisszene trägt sie die Züge einer mythischen Antigone-Figur. Sie muss ihrem Bruder die letzte Ruhestätte geben und bettet diesen auf Sonnenblumen inmitten eines Rapsfeldes. Danach fährt sie mit dem Rad nach Hause, wo sie zu ihrer großen Überraschung ihre Großeltern antrifft, die gerade von ihrer Mallorca-Reise zurückgekehrt sind und noch nicht wissen, was wirklich passiert ist. Für die unwissenden Großeltern wird sie zur Unheilverkünderin, zur Erzählerin der Katastrophe.

So wie bei Pausewang steht auch bei Wellenstein ein mutiges Mädchen im Zentrum der Geschichte. Lana ist das titelgebende sechzehnjährige „Mädchen von Tschernobyl“, da sie aus der Stadt Pripyat stammt, nur wenige Kilometer vom Atomkraftwerk entfernt. Als Sechsjährige wurde sie mit ihrer Mutter und ihrem Bruder nach Kiew evakuiert. Die real existierende Stadt Pripyat mit knapp 50.000 Einwohnern befindet sich ungefähr vier Kilometer vom Reaktor entfernt und wurde am 27. April 1986 evakuiert. Lanas Vater war Feuerwehrmann und bei den ersten Löscheinsätzen dabei. Er ist von diesem Löscheinsatz nie wieder nach Hause gekommen. Lana besucht ihre Großmutter, die in der evakuierten Zone lebt und den Tod ihres Sohnes nie wirklich verwunden hat. Sie pflegt dort ein kleines Mausoleum in Gedenken an ihren Sohn, dessen verstrahlter toter Körper auf Anordnung des Staates in einem Zinksarg weit weg in Moskau begraben wurde ${ }^{(36)}$. Sowohl im Text von Wellenstein wie auch bei Pausewang wird eine heile Kinderwelt zerstört. In beiden

34 P. Utz, Kultivierung der Katastrophe (Anm. 28), S. 15. These 4: Emotionalität, Solidarität und Dissonanz.

35 Ebd.

36 Diese Szene, wie auch die Figur der Großmutter, die nicht evakuiert werden wollte und allein ihr Leben in einem Dorf der kontaminierten Zone verbringt, ähnelt in gewissen Zügen den Berichten der Zeitzeuginnen Zinaïda Evdokimonva Kovalenka oder Anna Petrovna Badaïeva (Zonenbewohnerinnen ohne Genehmigung) in Alexijewitschs Text. 
Texten verlieren die Hauptfiguren einen Teil ihrer Familie durch die Katastrophe. Besonders wichtig wird dadurch in beiden Texten die besondere Beziehung der Enkel zur Großelterngeneration. Lana besucht ihre Großmutter heimlich an einem Wochenende, um das Grab ihres Vaters zu sehen. Am Grab verspürt sie nun nicht nur Traurigkeit, sondern auch eine große Wut:

\begin{abstract}
„À la tristesse se mêlait une noire colère. Pour venir ici, elle avait bravé les interdictions et les menaces, mais elle se sentait finalement bien impuissante devant cette petite croix fleurie. [...] Sans eux, sans les pompiers et sans les, liquidateurs' qui avaient circonscrit l'épanchement du geyser radioactif, toute l'Europe de l'Ouest aurait été dévastée, rongée par les radiations. Alors pourquoi les gens ne se souvenaient-ils pas de son père ? Pourquoi ne pensaient-ils jamais à Ivan Averine qui s'était porté avec courage au-devant des flammes, ni à ses enfants orphelins? Sans eux, la Zone d'exclusion n'aurait pas fait trente kilomètres... mais huit cents!“ (Wellenstein, S. 21-22).
\end{abstract}

Lana trifft bei diesem Besuch auf Wildpferde - auf Przewalskipferde, die offenbar aus einem Nationalpark stammen und zu Forschungszwecken in die kontaminierte Zone gebracht wurden. Die Wildpferde - anarchisch-vorzivilisatorische Naturgeschöpfe - stehen für die Kraft der Natur, die in diesen zerstörten Räumen überleben können und überlebensfähiger sind als die Menschen. Sie stehen aber vor allem für Lanas Beziehung zu ihrem verstorbenen Vater. Lana will diese Pferde vor Wilderern schützen und kehrt bei diesem Abenteuer in ihre Geburtsstadt Pripyat zurück, die zur Geisterstadt geworden ist. Der Begriff „Geisterstadt“ ist hier wörtlich zu nehmen, da Lana auf Geister der Vergangenheit stößt, auf Geister der Toten von Tschernobyl: ihren Vater und die verstorbene Schwester eines jungen Mannes und Photographen Sascha -, den sie in dieser toten Stadt trifft und der ihr hilft. Auch er und seine kleine Schwester, die an Leukämie starb (Wellenstein, S. 131), sind in Pripyat aufgewachsen, bis es zur Katastrophe kam. Er wird mit seinen Bildern die Zerstörung festhalten und für Lana und seine verstorbene Schwester die Aufklärungsarbeit über die (vertuschten) Ausmaße der Katastrophe leisten:

„- Bien sûr, acquiesça Sacha. Tu sais qu'ils minimisent le nombre de victimes, n'est-ce pas? Mais tu connais le chiffre exact? Vas-y. Dis un chiffre.

Je ne sais pas, reconnut-elle.

Cinquante-six victimes.

Elle émit un rire sans joie... [...]

Il se tortilla pour sortir un carnet de notes de la poche de son jean et en feuilleta les pages.

- J'estime que 25000 personnes sont mortes suite à leur intervention à Tchernobyl. Plus de 160000 personnes sont considérées comme invalides ou malades. Enfin, plus de cinq millions de personnes vivent sur des territoires contaminés et n’ont pas les moyens de déménager, ni de se soigner. [...]

- Je veux que les gens sachent. Qu'ils se souviennent. Je suis convaincu qu'aujourd'hui, si un nouvel accident avait lieu quelque part dans le monde, au sein d'une autre centrale nucléaire, il se passerait exactement la même chose qu’en 1986. La vérité serait jetée au fond d'un trou et la souffrance des victimes étouffée. Tu trouves ça normal ?"(Wellenstein, S. 96 und 131$)^{(37)}$.

37 Die Opferzahlen schwanken in der Tat nach wie vor zwischen Extremen von ca. 30 Toten über eine Million. Cf. Galia Ackerman, Tchernobyl. Retour sur un désastre, Paris, Folio, 2007. 
Während der Verfolgungsjagd mit den Wilderern wird Lana von einer Kugel getroffen und verletzt. Diese Verletzung provoziert einen Flashback und Lana durchlebt erneut den traumatischen Tag und die Nacht des 26. Aprils 1986, an dem sie ihren Vater verlor und evakuiert wurde. Wie Janna-Berta in Die Wolke entscheidet sich auch Lana dazu, in die kontaminierte Zone zurückzukehren - sie macht in gewissem Sinn eine Reise in die Unterwelt, in die Welt der Zerstörung und Bedrohung. Diese Reise ist für sie notwendig, um ihre Traumata zu verarbeiten und darauf aufbauend vielleicht einen neuen Weg einzuschlagen. Ebenso wie Pausewangs Protagonistin wird auch Wellensteins Heldin gegen das Vergessen anschreiben ${ }^{(38)}$. Sie will alles von ihrer Großmutter über die Katastrophe von Tschernobyl erfahren und es dann niederschreiben:

„-Tu vas tout me raconter, dit-elle à sa Babouchka.

Tout ce dont tu te souviens, et je vais écrire ton histoire. J'irai voir tes voisins. Je parlerai à tout le monde, ici et ailleurs, à Kiev, et plus loin. Sacha prendra des photos, pour fixer les visages, et moi, j'immortaliserai les voix. Je vais tout dire. Nous allons faire témoigner les gens. [...]“ (Wellenstein, S. 158).

\section{Die zerstörte und versehrte Herkunftswelt und das post-apokalyptische Setting}

Bei Pausewang ist die Katastrophe immanent. Die Hauptfigur muss sich in der Katastrophe bewähren. Sie muss allein Entscheidungen treffen und vor der gefährlichen Wolke fliehen. Die Flucht wird zum Albtraum, da die Katastrophe das Land in ein Chaos verwandelt. Die gewohnten Regeln des Zusammenlebens gelten nicht mehr. Es herrscht Massenpanik. Im Auto hört Janna-Berta von der Schließung der Grenze zur DDR, wo man keine Flüchtlinge aufnehmen will:

„Wer die Autobahn in Richtung Eisenach befahren hatte, war darauf aus gewesen, sich nach Berlin oder in die DDR zu retten. Aber nun war seit einer Stunde die Grenze von östlicher Seite geschlossen worden. Ein schwerer Lastwagen hatte daraufhin die ostdeutschen Schlagbäume weggedrückt, um den PKW-Kolonnen hinter ihm freie Durchfahrt zu verschaffen. Aber die Grenzsoldaten hatten sie mit Maschinenpistolen aufgehalten. Die Wagen, die noch hatten wenden können, waren wieder in den Westen zurückgeflüchtet. [...] ,Mörder!', schrie jemand. ,Schießen auf ihre Brüder!', Die sind genauso in Panik wie wir', sagte der Blonde ruhig. ,Außerdem wird bei uns auch geschossen. Und ich wette, das ist alles erst der Anfang. Aus dem Absperrungsgürtel um Schweinfurt kommt keiner mehr lebend raus. Wenn ihn die Radioaktivität nicht umbringt, dann das Militär. Die werden die stark Verseuchten mit Gewalt daran hindern, sich unter die Davongekommenen zu mischen. ',Du spinnst', rief die Sommersprossige. ,Die können die Leute doch nicht wie die Hasen abknallen.“' (Pausewang, Die Wolke, S. 75).

Janna-Bertas Eltern, zwei ihrer Geschwister und ihre Großmutter befinden sich im Zentrum der Katastrophe. Das Mädchen weiß lange nicht, was mit ihren Familienangehörigen passiert ist und wie es ihnen geht. Das ist das Schlimmste an ihrer Situation zusätzlich zu ihren Schuldgefühlen am Unfalltod ihres kleinen Bruders Uli. Die Katastrophe erzeugt eine Kettenreaktion an menschlicher Gewalt: der Autofahrer, der Uli tötet, bleibt nicht stehen. Die Menschen denken nur ans eigene Überleben.

38 Swetlana Alexijewitsch wiederum schreibt am Ende ihres eigenen Zeugenberichts: „Je voyageais, je parlais, je notais. [...] Plus d'une fois, j'ai eu l'impression de noter le futur“, in: S. Alexievitch, $L a$ supplication (Anm. 1), S. 33. 
Mitmenschen, die eine Gefahr darstellen, werden ausgegrenzt und gemieden. Wie Peter Utz betont, bricht in der Katastrophe ein System, eine Ordnung in sich zusammen ${ }^{(39)}$. Die anarchische Kraft der Katastrophe zeigt sich im Unvermögen, der Katastrophe Herr zu werden sowie in einem Ausmaß menschlicher Gewalt, das davor unvorstellbar schien. Pausewang betont regelrecht das menschliche Versagen nach der Katastrophe in den eindringlichen Fluchtszenen und am Nicht-Funktionieren der Katastrophenschutzpläne.

Auch im Roman von Wellenstein geht eine Welt unter. Bereits im Paratext auf Seite zwei vor dem ersten Kapitel wird in einem kurzen faktualen Informationstext die Katastrophe von Tschernobyl dargestellt und dabei vor allem die Zerstörung und Verschmutzung der Erde für hunderte von Jahren betont:

„Le 26 avril 1986, à Tchernobyl, en Ukraine, a eu lieu la première grande catastrophe nucléaire de l'histoire, faisant des milliers de victimes et polluant pour des siècles les terres alentour. La zone d'exclusion alors mise en place autour de la centrale, destinée à tenir la population à l'écart des territoires les plus radioactifs, reste aujourd'hui abandonnée et ne sera plus habitable avant plusieurs centaines d'années“ (Wellenstein, S. 2).

Zehn Jahre nach der Katastrophe kehrt Lana in die verseuchte Zone zurück. Diese Zone blieb nicht unbewohnt. Fauna und Flora haben sich dort weiterentwickelt und den Raum eingenommen.

„Tout en tripotant le dosimètre dans sa poche, la jeune fille entra dans le cimetière. Ici encore, la nature avait envahi l'espace. L'herbe proliférait dans les allées. De la mousse verdissait les pierres tombales. Il n'y avait plus de bouquets, mais des dizaines de fleurs sauvages, jaunes, bleues et blanches, rendaient hommage aux disparus. Des champignons poussaient également ça et là en grappes visqueuses“ (Wellenstein, S. 20).

Lana stößt auf fette Hirsche, die nicht mehr gejagt werden, auf Wildenten und, wie bereits erwähnt, vor allem auf Wildpferde. Die Natur, Pflanzen und Tiere geben ihr Kraft und Mut in Momenten der Verzweiflung und Wut angesichts des Todes ihres Vaters. Gleichzeitig stellt sie die Verformungen an der Natur fest. Sie findet beispielsweise mutierte Schwalbeneier (Wellenstein, S. 26) und ist sich der unsichtbaren Bedrohung der radioaktiven Verseuchung bewusst:

„D'un air morne, la jeune fille regarda autour d'elle la nature luxuriante. La mort coulait dans ces terres fertiles. Les racines des arbres, leur sève, l'eau des rivières, tout ici charriait sans un bruit la menace de l'atome devenu fou. Le lait, le blé, les framboises, les fraises de Tchernobyl avaient apporté leur lot de maladies, cancers, leucémies... Tout ça à cause des mensonges d'autorités frileuses ou cupides" (Wellenstein, S. 26).

Die Heimkehr und Rückkehr in das Land nach der Katastrophe ist Teil der Katastrophenerzählung. Das Trauma der Evakuierung und der Vertreibung braucht zu seiner Verarbeitung offenbar die Rückkehr in die zerstörte Welt.

In ähnlicher Weise findet die Verarbeitung der Atomkatastrophe in dem aktuellen Roman Heimkehr nach Fukushima von Adolf Muschg statt, der eine Liebesgeschichte in der kontaminierten Zone von Fukushima erzählt. In diesem Roman, der allerdings nicht zur KJL zählt, ist die unsichtbare Zerstörung der Landschaft ebenfalls ein zentrales Element. Eine der Hauptfiguren, die den zynischen Kommentar hinsichtlich 
des Geschehenen übernimmt, stellt dem katastrophalen Zustand der Landschaft den Begriff der Idylle gegenüber, beides „typische“ ökokritische Gattungsmuster ${ }^{(40)}$.

„Wir stehen vor einem japanischen Kunstwerk der Verzweiflung, einem flächendeckenden Tagebau des reinigenden Wahns.

Aber auch was nicht, was nie wieder gesäubert werden kann, Busch und Wald, Baum und Gras, Berg und Tal: alles blüht und gedeiht, wuchert und wildert in diesem Silent Spring, je naturbelassener, desto üppiger. Fukushima ist schon fast beängstigend malerisch. [...] War was? Hier und jetzt leuchtet sie von jungem Grün, die verstrahlte Erde, und wenn Fukushima immer von der Natur gesegnet war, jetzt hat es sich fast zu einer fast atemberaubenden Idylle ausgewachsen. Darum atmet man ja auch besser durch eine Maske, wenn man sich nicht den Tod holen will. Aber was soll's? Ein entsprechender Befund wird sich, statistisch gesehen, erst in zwanzig, dreißig Jahren zeigen. Und in alter Zeit, der großen Zeit der Idylle, sind die Menschen nicht einmal so alt geworden“(41).

\section{Erinnerungsarbeit nach der Katastrophe}

Gudrun Pausewang wurde häufig Angstmacherei vorgeworfen. In einem Zeit-Artikel wird sie gar als „Lehrerin der Angst“ bezeichnet ${ }^{(42)}$. Darauf reagiert sie im Nachwort zum Roman von Noch lange danach (2012) folgendermaßen:

„Ich habe dieses vorliegende kleine Buch ,Noch lange danach“ wie auch ,Die Wolke` nicht geschrieben aus Lust, Angst zu erzeugen. (Wobei ich bei dieser Gelegenheit darauf hinweisen möchte, dass man Angst nicht so verteufeln sollte. Die Fähigkeit, Angst zu empfinden, wurde uns von der Natur mitgegeben als Hilfe zum Überleben. Wären wir Menschen nicht imstande, Angst zu empfinden, gäbe es unsere Gattung schon längst nicht mehr!). [...] Ich schrieb beide Bücher als Warnung. [...] Ich wünsche mir, dass der Leser, gleichgültig, ob Jugendlicher oder Erwachsener, durch den Schock des Inhalts hindurchfindet zu der Frage, die er sich selbst stellt: Womit kann ich im Rahmen meiner Möglichkeiten tätig dazu beitragen, dass das, was hier fiktiv als Reaktorkatastrophe in Deutschland geschildert wurde, nie Realität wird“(43).

Nach dem Reaktorunfall in Fukushima 2011 haben Pausewangs Texte eine erneute Aktualität erlangt und ihre Warnung hat sich als gerechtfertigt erwiesen. Die Katastrophe von Tschernobyl, die viele bereits schon vergessen hatten und vergessen wollten, wurde wieder tragisch in Erinnerung gerufen. Für Uwe Jahnke, der eine Biographie Pausewangs verfasst und die Medienauftritte der Autorin unmittelbar nach der Katastrophe in Fukushima analysiert hat, wird deutlich, „dass es der Autorin grundsätzlich weniger um die Verbreitung von Ängsten besonders unter Jugendlichen geht, als vielmehr darum zu warnen und auch Jugendliche dazu zu bewegen, in den politischen Diskurs einzugreifen und sich auf diese Weise für die eigene Zukunft zu interessieren und wenn möglich zu engagieren“(44). Pausewangs Hauptfigur Janna-Berta verkörpert

40 Cf. E. Zemanek (Hg.), Ökologische Genres (Anm. 4), Kapitel „Idylle“, S. 73 sq. und Kapitel „Literarische Umwelt-Risikonarrative", S. 211 sq.

41 Adolf Muschg, Heimkehr nach Fukushima, München, Beck, 2018.

42 www.zeit.de/2004/02/Pausewang_02 (09.09.2019).

43 Gudrun Pausewang, „Nachwort“, in: Noch lange danach, Ravensburg, Ravensburger Buchverlag, 2012, S. 124-125.

44 Uwe JAhnke, www.kids-media.uzh.ch/1-2013/jahnkedefinitiv.pdf (15.11.2019). 
diese Art von engagierter Widerspenstigkeit. Als sie von ihrer Tante im Krankenhaus abgeholt wird, wird sie von dieser aufgefordert, eine Mütze zu tragen, was Janna-Berta jedoch ablehnt und mit ihrer Erinnerungspflicht argumentiert:

„Ich würde sie aufsetzen', sagte Helga mit einer senkrechten Falte zwischen den Brauen. ,Viele Leute reagieren komisch, wenn sie merken, dass jemand aus der verseuchten Gegend kommt. Es gibt sogar Hotels, die den Evakuierten Unterkunft verweigern, wenn - wenn die Krankheit deutlich sichtbar ist. Sie sagen, das vertreibt ihnen die Kundschaft.' ,Ich verstehe', sagte Janna-Berta hart. ,Sie wollen nicht erinnert werden. 'Wie gesagt, ich würde die Mütze aufsetzen', sagte Helga.

Janna-Berta griff nicht nach der Mütze. ,Ich will sie aber daran erinnern', sagte sie" (Pausewang, Die Wolke, S. 126).

Der Jugendbuchforscher Malte Dahrendorf behauptet, und dabei würde ich ihm zustimmen, dass Gudrun Pausewang einer Literatur verpflichtet sei, die „in der Tradition der Aufklärung steht, die aufklären, gesellschaftlich Verdrängtes aufdecken will. Aber noch mehr: Diese Literatur ist eine operative Literatur. [...] Gudrun Pausewang schildert nicht nur Katastrophen, nicht nur unhaltbare Verhältnisse, sondern zugleich Möglichkeiten des Handelns, der handelnden Gegenwehr “(45). Für den Literaturwissenschaftler Peter Utz ist die Warnung in jedem Fall eine literarische Antwort auf die Katastrophe, eine künstlerische Form der Einschreibung ins kulturelle Gedächtnis:

„Sie [die Literatur, H.H.] horcht in den individuellen und gesellschaftlichen Untergrund hinein und artikuliert die ,Unruhe', wenn die Ruhe als erste Bürgerpflicht gilt. Und sie weckt eine ökologische Aufmerksamkeit für die ,Natur““(46).

Ähnliches gilt auch für Wellenstein. Auch ihr Roman schreibt sich in die Tradition der Aufklärung ein. Sowohl Janna-Berta als auch Lana übernehmen am Ende ihrer Geschichte die Handlungsmacht. Beide Texte tragen utopische Züge in der Hinsicht, wie Gabriele von Glasenapp sie beschreibt und Greta Thunberg sie vielleicht verkörpert, nämlich in der Hoffnung auf eine imaginierte Veränderbarkeit der Welt durch jugendliche Akteure und Akteurinnen, in der Hoffnung auf eine bessere, schönere und heilere Welt ${ }^{(47)}$.

\section{Zusammenfassung}

Die deutschsprachige Kinder- und Jugendliteratur hat vergleichsweise früh „Klassiker der ökokritischen Literatur" hervorgebracht, die die Gefahr der Atomwaffennutzung und das Szenario eines möglichen Supergaus ins Zentrum der Handlung stellen. Einen besonderen Platz nimmt hier Gudrun Pausewangs Bestseller Die Wolke. Jetzt werden wir nicht mehr sagen können, wir hätten von nichts gewusst ein, der ein Jahr nach der atomaren Katastrophe von Tschernobyl im April 1986 erschienen ist. Dieser Roman wird verglichen mit einem jüngeren französischen Jugendroman, Aurélie Wellensteins

45 Malte Dahrendorf, „Wider das Verdrängen und Verharmlosen. Gudrun Pausewangs Atomkatastrophenbücher“, kjl\&m, 61/4 (2009), S. 23-26 (Themennummer: „Die angekündigte Katastrophe oder: KJL und Umweltschutz"). 
La fille de Tchernobyl (2016), der wiederum intertextuell stark von Swetlana Alexijewitschs Dokumentarroman Tschernobyl. Eine Chronik der Zukunft (1997) geprägt ist. Welche narrativen Besonderheiten weisen diese Katastrophendarstellungen in der Kinder- und Jugendliteratur auf? Im ersten Teil des Beitrags werden die weiblichen Hauptfiguren der zwei Jugendromane vorgestellt und dabei auch auf die Verknüpfung von faktualem und fiktionalem Modus eingegangen. Im zweiten Teil werden die zerstörten Räume analysiert und im abschließenden Teil soll gezeigt werden, inwieweit diese Romane Erinnerungsarbeit leisten und gleichzeitig Aufklärung einfordern. Anders gesagt, soll es um die Frage nach den erzieherischen Absichten dieser Texte gehen. Sind sie reine "Angstmacherei", wie es Gudrun Pausewang vorgeworfen wurde, oder hat das Setting der "Katastrophe“, das nicht neu ist, sondern eine lange literarische Tradition hat und in der internationalen Umweltbewegung eine zentrale Rolle spielt, noch andere Funktionen?

\title{
Résumé
}

La littérature de langue allemande pour enfants et adolescents a produit relativement tôt des "classiques de la littérature écocritique», qui ont placé le danger de l'emploi d'armes nucléaires et le scénario d'un éventuel accident nucléaire au centre de leur trame narrative. Le best-seller de Gudrun Pausewang Le nuage, publié un an après la catastrophe nucléaire de Tchernobyl en avril 1986, occupe une place particulière ici. Ce texte est comparé à un roman français pour la jeunesse, La fille de Tchernobyl d'Aurélie Wellenstein (2016), qui, à son tour, est fortement imprégné du roman documentaire La supplication (1997) du Prix Nobel Svetlana Alexievitch. Quelles sont les particularités narratives de ces représentations de la catastrophe dans la littérature pour enfants et adolescents? Dans la première partie de notre contribution, nous présentons les personnages principaux féminins des deux romans et abordons également l'enchevêtrement du fictionnel et du factuel. Dans la deuxième partie, les espaces détruits sont analysés et, dans la dernière partie, il s'agit de montrer dans quelle mesure ces romans font un travail de mémoire et demandent en même temps des comptes. En d'autres termes, il s'agit d'interroger les intentions pédagogiques de ces textes: sont-ils purement "alarmistes", comme on le reprochait à Gudrun Pausewang, ou bien le cadre de la "catastrophe», qui n'est pas nouveau mais repose sur une longue tradition littéraire, et joue par ailleurs un rôle central dans le mouvement environnemental international, remplit-il d'autres fonctions?

\begin{abstract}
The German-language literature for children and adolescents produced "classics of ecocritical literature" comparatively early on. This sub-genre has been placing the dangers pertaining to nuclear weapons and the scenario of a potential nuclear catastrophe at the heart of its narratives. Gudrun Pausewang's bestseller The Cloud. Now we will no longer be able to say that we did not know about anything, which was published one year after the Chernobyl nuclear disaster in April 1986, holds a central place here. This novel is compared to a more recent French youth novel, Aurélie Wellenstein's La fille de Tchernobyl (2016), which in turn is strongly influenced by Svetlana Alexievitch's documentary novel Chernobyl Prayer. A chronicle of the Future (1997). What are the narrative features of these representations of catastrophes in children's and young people's literature? In the first part of my contribution, I introduce the main female
\end{abstract}


characters of the two youth novels and I address the combination of factual and fictional modes. Then, I analyse the spaces of destruction; in the final part, I demonstrate to what extent these novels function as works of remembrance while simultaneously requiring educational clarification. In other words, I aim to explore the pedagogical goals of these works. Should they be considered as pure "scaremongering" (of which Gudrun Pausewang was accused); or does the narrative context of the "catastrophe" - a concept with a long literary tradition which has been playing a central role in the international environmental movement - have other functions? 